\title{
PI3K signaling in B cell and T cell biology
}

\author{
Klaus Okkenhaug ${ }^{1}{ }^{*}$, Martin Turner $^{1}$ and Michael R. Gold ${ }^{2}$ \\ ' Laboratory of Lymphocyte Signalling and Development, Babraham Institute, Cambridge, UK \\ ${ }^{2}$ Department of Microbiology and Immunology, University of British Columbia, Vancouver, BC, Canada \\ *Correspondence: klaus.okkenhaug@babraham.ac.uk \\ Edited and reviewed by: \\ Ellis L. Reinherz, Dana-Farber Cancer Institute, USA
}

Keywords: PI3K/AKT/mTOR, PI3K pathway inhibitors, T cell, B cell, signal transduction

The drug Idelalisib is the first PI3K inhibitor to be approved by the FDA for clinical use. It is therefore timely to take stock of our current understanding of the role of PI3Ks in the immune system. The phosphoinositide 3-kinases (PI3Ks) control many key functions in immune cells (1). PI3Ks phosphorylate PtdIns $(4,5) \mathrm{P}_{2}$ to yield PtdIns $(3,4,5) \mathrm{P}_{3}$, which acts as a second messenger signaling molecule that controls the activation of Akt and other proteins with PH domains. Initially, PI3K inhibitors such as Wortmannin, LY294002, and Rapamycin were used to establish a central role for PI3K pathway in immune cells. More recent progress in understanding the role of this pathway in cells of the immune system has been made through the generation of wide range of gene-targeted mouse models as well as with the development of highly selective small molecule inhibitors, culminating in the FDA approval of the PI3K $\delta$ inhibitor Idelalisib in 2014. Together, lab experiments, preclinical, and clinical trials have revealed how PI3Ks control B cell and T cell development, $\mathrm{T}$ helper cell differentiation, regulatory $\mathrm{T}$ cell (Treg) development and function, B cell and $\mathrm{T}$ cell trafficking, immunoglobulin class switching, and much more.

The class I PI3Ks are heterodimers composed of a catalytic subunit and a regulatory subunit. The $\mathrm{p} 110 \alpha, \mathrm{p} 110 \beta$, and $\mathrm{p} 110 \delta$ catalytic subunits form heterodimers with either of the p $85 \alpha, \mathrm{p} 55 \alpha$, $\mathrm{p} 50 \alpha, \mathrm{p} 85 \beta$, or $\mathrm{p} 55 \gamma$ regulatory subunits, each of which contains SH2 domains that are engaged by tyrosine phosphorylated proteins. By contrast, the p110 $\gamma$ catalytic subunit forms heterodimers with either the $\mathrm{p} 84$ or p101 regulatory subunits that are recruited to the $\mathrm{G} \beta \gamma$ subunits released by G-protein coupled receptors. The respective heterodimers are often referred to as $\mathrm{PI} 3 \mathrm{~K} \alpha, \mathrm{PI} 3 \mathrm{~K} \beta$, $\mathrm{PI} 3 \mathrm{~K} \delta$, or PI $3 \mathrm{~K} \gamma$, respectively. Progress has been made in understanding the often non-redundant roles of each of these PI3K isoforms in immunity (2). The majority of these efforts have been directed at $\mathrm{PI} 3 \mathrm{~K} \delta$ and $\mathrm{PI} 3 \mathrm{~K} \gamma$, which are highly expressed in cells of the immune system.

mTOR inhibitors such as rapamycin are already established as among the most commonly used drugs to prevent transplant rejection and are increasingly also evaluated for the use in cancer (3). Most recently, the PI3K $\delta$-selective inhibitor Idelalisib (CAL-101) has received FDA approval for the treatment of chronic lymphocyte leukemia and indolent non-Hodgkin's lymphoma following on from successful clinical trials showing dramatic improvements in progress-free survival $(4,5)$. Many other PI3K inhibitors are going through clinical trials with the aim to treat cancers, and inflammatory and autoimmune diseases.
This eBook contains 11 chapters that cover different roles of the PI3K and mTOR pathways, primarily in B cells and T cells. Limon and Fruman review the role of PI3Ks in B cell development and activation and also consider how the related Akt, Foxo, and mTOR pathways affect B cell biology (6). Marshall and colleagues present a complementary view, focusing on the different mechanisms of activation of PI3K in B cells, and the roles of the different phosphatases as well as individual role for additional $\mathrm{PIP}_{3}$ effectors such as Bam32, Tapp1, and Tapp2 (7). Puri and Gold pick up on this theme and further explore the clinical utility of blocking PI3K activity in B cells, both in the context of autoimmune diseases, but also by summarizing the early clinical trials that led to accelerated approval of Idelalisib for the treatment of B cell lymphomas (8). Bhatt and Damania consider the roles of PI3K and mTOR in the transformation of B cells by Kaposi sarcoma viruses and how PI3K and/or mTOR inhibitors should be considered for the treatment of virally induced lymphomas (9).

Lewings and colleagues consider how PI3K signaling strength affects $\mathrm{T}$ cell differentiation events. Their data show that high levels of PI3K signaling can antagonize the differentiation of Treg in favor of the differentiation of effector T cells (10). Okkenhaug and colleagues present a somewhat different perspective, reviewing evidence that low PI3K activity is also detrimental to Treg function and attempt to reconcile some of the different conclusions regarding the role of PI3K in Treg function (11). Newton and Turka consider the role of the $\mathrm{PIP}_{3}$ phosphatase Pten in T cells and how unrestrained PI3K signaling can lead to T cell transformation (12). They further discuss the differential roles of Pten on PI3K signaling and genome stability. Gamper and Powell discuss data that challenge the common assumption that PI3K and mTOR lie on a common pathway, by providing a detailed discussion of similarities but importantly also key differences in the phenotypes observed when either PI3K or mTOR is inhibited in CD4 T cells (13). Similarly, Finlay discusses recent data showing that the PI3K is in fact dispensable for mTOR activation in CD8 T cells and that a Pdk1-mTOR axis regulates T cell metabolism independently of PI3K and Akt (14).

These studies indicate that we need to start considering other signaling pathways regulated by PI3K. Venigalla and Turner summarize recent evidence suggesting a key role for PI3K, often in concert with p38, in regulating mRNA stability and translation (15). Some of these effects may also be mediated by mTOR.

Drugs against PI3K have become a clinical reality. However, not all PI3K isoforms can be targeted as specifically as Idelalisib inhibits 
PI3K $\delta$. Blunt and Ward summarized the progress in developing PI3K inhibitors, with particular focus on Idelalisib, but then go on to summarize the roles of the $\mathrm{PIP}_{3}$ 5-phosphatases SHIP1 and SHIP2 (16). Moreover, they review the development of allosteric drugs that activate SHIP as an alternative strategy to inhibit PI3K signaling in immune cells.

Altogether, these reviews summarize the remarkable progress in our understanding of how PI3Ks regulate many facets of the adaptive immune response, but also help highlight many unresolved and even controversial areas of research. We are grateful for the considerable efforts that the authors have made to help us compile this ebook for Frontiers in Immunology.

\section{REFERENCES}

1. Okkenhaug K. Signaling by the phosphoinositide 3-kinase family in immune cells. Annu Rev Immunol (2013) 31:675-704. doi:10.1146/annurev-immunol032712-095946

2. Okkenhaug K. Rules of engagement: distinct functions for the four class I PI3K catalytic isoforms in immunity. Ann N Y Acad Sci (2013) 1280:24-6. doi: $10.1111 /$ nyas. 12027

3. Laplante M, Sabatini DM. mTOR signaling in growth control and disease. Cell (2012) 149:274-93. doi:10.1016/j.cell.2012.03.017

4. Furman RR, Sharman JP, Coutre SE, Cheson BD, Pagel JM, Hillmen P, et al. Idelalisib and rituximab in relapsed chronic lymphocytic leukemia. $N$ Engl J Med (2014) 370:997-1007. doi:10.1056/NEJMoa1315226

5. Gopal AK, Kahl BS, De Vos S, Wagner-Johnston ND, Schuster SJ, Jurczak WJ, et al. PI3Kdelta inhibition by idelalisib in patients with relapsed indolent lymphoma. N Engl J Med (2014) 370:1008-18. doi:10.1056/NEJMoa1314583

6. Limon JJ, Fruman DA. Akt and mTOR in B cell activation and differentiation. Front Immunol (2012) 3:228. doi:10.3389/fimmu.2012.00228

7. Pauls SD, Lafarge ST, Landego I, Zhang T, Marshall AJ. The phosphoinositide 3-kinase signaling pathway in normal and malignant B cells: activation mechanisms, regulation and impact on cellular functions. Front Immunol (2012) 3:224. doi:10.3389/fimmu.2012.00224

8. Puri KD, Gold MR. Selective inhibitors of phosphoinositide 3-kinase delta: modulators of B-cell function with potential for treating autoimmune inflammatory diseases and B-cell malignancies. Front Immunol (2012) 3:256. doi:10.3389/ fimmu.2012.00256
9. Bhatt AP, Damania B. AKTivation of PI3K/AKT/mTOR signaling pathway by KSHV. Front Immunol (2012) 3:401. doi:10.3389/fimmu.2012.00401

10. Han JM, Patterson SJ, Levings MK. The role of the PI3K signaling pathway in CD4(+) T cell differentiation and function. Front Immunol (2012) 3:245. doi:10.3389/fimmu.2012.00245

11. Soond DR, Slack EC, Garden OA, Patton DT, Okkenhaug K. Does the PI3K pathway promote or antagonize regulatory $\mathrm{T}$ cell development and function? Front Immunol (2012) 3:244. doi:10.3389/fimmu.2012.00244

12. Newton RH, Turka LA. Regulation of T cell homeostasis and responses by Pten. Front Immunol (2012) 3:151. doi:10.3389/fimmu.2012.00151

13. Gamper CJ, Powell JD. All PI3Kinase signaling is not mTOR: dissecting mTORdependent and independent signaling pathways in T cells. Front Immunol (2012) 3:312. doi:10.3389/fimmu.2012.00312

14. Finlay DK. Regulation of glucose metabolism in T cells; new insight into the role of Phosphoinositide 3-kinases. Front Immunol (2012) 3:247. doi:10.3389/ fimmu.2012.00247

15. Venigalla RK, Turner M. RNA-binding proteins as a point of convergence of the PI3K and p38 MAPK pathways. Front Immunol (2012) 3:398. doi:10.3389/ fimmu.2012.00398

16. Blunt MD, Ward SG. Pharmacological targeting of phosphoinositide lipid kinases and phosphatases in the immune system: success, disappointment, and new opportunities. Front Immunol (2012) 3:226. doi:10.3389/fimmu.2012. 00226

Conflict of Interest Statement: The authors declare that the research was conducted in the absence of any commercial or financial relationships that could be construed as a potential conflict of interest.

Received: 22 September 2014; accepted: 20 October 2014; published online: 03 November 2014.

Citation: Okkenhaug K, Turner M and Gold MR (2014) PI3K signaling in B cell and T cell biology. Front. Immunol. 5:557. doi: 10.3389/fimmu.2014.00557

This article was submitted to T Cell Biology, a section of the journal Frontiers in Immunology.

Copyright (C) 2014 Okkenhaug, Turner and Gold. This is an open-access article distributed under the terms of the Creative Commons Attribution License (CC BY). The use, distribution or reproduction in other forums is permitted, provided the original author(s) or licensor are credited and that the original publication in this journal is cited, in accordance with accepted academic practice. No use, distribution or reproduction is permitted which does not comply with these terms. 